\section{Diferentes propuestas para la representación geométrica de edificios históricos}

Irantzu Álvarez González, Ane Lopetegl Galarraga, Amaia Mesanza Moraza, Álvaro Rodríguez Miranda, José Manuel Valle Melón, IRATXe Vicente Espina

Lab. Doc. Geométrica del Patrimonio

Unidad Asociada CSIC/Universidad del País Vasco. Grupo de Arqueología

Tardoantigua y Medieval. Arqueología de la Arquitectura (GATMAA)

\section{Resumen}

La documentación geométrica y la representación de edificios con el objetivo de servir como base a estudios históricos o arquitectónicos ofrece una variada oferta metodológica, cada una de ellas con unas características específicas. Antes de acometer una documentación, es necesario realizar un análisis previo de las necesidades finales del trabajo para elegir el método de documentación más conveniente.

Palabras Clave: Topografía, fotogrametría, modelos virtuales, documentación geométrica.

\section{Abstract \\ Geometric documentation and the representation of buildings, with the objective of using these as a basis for historic or architectonic studies, offer a wide range of methodologies, each with their own specific characteristics. Before beginning documentation, it is necessary to make a preliminary analysis of the final needs of the work to choose the most suitable method of documentation.}

Key words: Surveying, photogrammetry, virtual models, geometric documentation.
En la mayoría de las ocasiones la documentación de un edificio entraña una alta complejidad, bien por sus características, bien por la forma de llevar a cabo su representación. Esta dificultad se amplía aún más, cuando se trata de un edificio de carácter histórico ya que los detalles que de una manera u otra hacen de él un elemento de singular interés deben quedar claramente definidos. De igual manera, si sobre este edificio se va a realizar algún tipo de análisis especial que requiera una documentación geométrica sobre la que representarse o como base o ayuda al mismo análisis, la representación deberá cumplir los requisitos necesarios para dicho estudio.

Actualmente, existe una gran diversidad de técnicas topográficas-cartográficas con aplicaciones a la documentación de edificios históricos, por lo tanto, cuando se requiere contar con documentación geométrica no es necesario que se limite exclusivamente a una colección de plantas y alzados obtenidos por restitución fotogramétrica. Al contrario, el método de documentación más adecuado a cada caso concreto parte de un estudio previo de las necesidades de registro, de los análisis que vayan a realizarse basados en los datos geométricos y de las representaciones gráficas que quieran obtenerse.

A este respecto, se describirán algunos métodos de documentación que se han utilizado y en alguna medida desarrollado en el Laboratorio de Documentación Geométrica del Patrimonio, mostrando ejemplos y enumerando las características de cada uno. Por otro lado, estos productos obtenidos de la documentación no son incompatibles entre sí, de hecho es posible obtener productos mixtos que comparten las características de varios métodos, como se muestra en la figura 1.

En un estudio histórico o arquitectónico el edificio se concibe como un elemento único y tridimensional, por lo tanto, el modelo gráfico que lo represente cumplirá estas dos características: unicidad y tridimensionalidad, frente a las representaciones como conjuntos de alzados independientes entre sí y con los cuales es difícil relacionar las diferentes partes de un mismo edificio.

La forma más simple de representación es mediante la realización de un modelo volumétrico consistente en la extracción de las líneas definitorias más importantes del edificio, empleando para ello topografía clásica. Esta representación pese a su sencillez, no ha sido posible realizarla de una forma ágil y rápida hasta hace relativamente poco tiempo, con la llegada de los sistemas de medida directa de distancias incorporados en las estaciones topográficas. Anteriormente, la dificultad de acceso a los puntos, junto con la lentitud de los métodos de captura topográfica desembocaba en el empleo de otras técnicas de representación. 


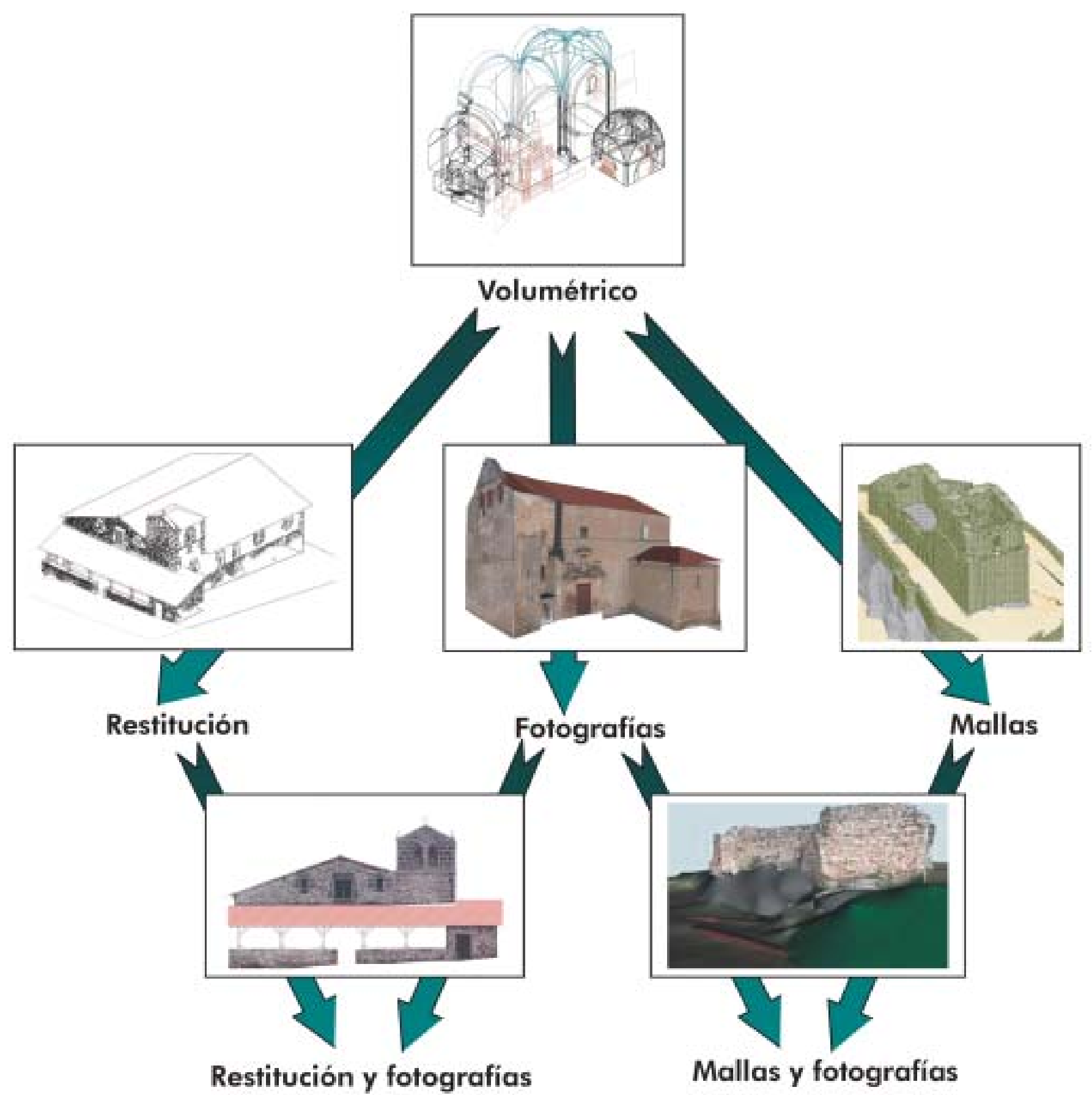

Fig. 1. Posibilidades de documentación tridimensional de un edificio histórico:

-Iglesia de la Asunción de Viñaspre (Lanciego, Álava): volumétrico y modelo virtual obtenido a partir de imagen fotográfica.

- Iglesia de San Andrés de Astigarribia (Mutriku, Guipúzcoa): restitución y restitución con imagen fotográfica de fondo.

-Castillo de Lanos (Ocio, Álava): mallas y mallas con textura fotográfica. 
Fig. 2. Volumétrico alámbrico y sólido de la ermita de la Vera Cruz (Durango, Bizkaia)

Como las necesidades que debe cubrir la representación final pueden ser muy variadas, será necesario definir previamente varios aspectos:

-El grado de detalle que se quiere alcanzar, es decir, definir cuál va a ser la unidad mínima que debe aparecer representada y que coincidirá con la unidad mínima que tenga sentido dentro del estudio temático que vaya a acometerse. De esta forma se podrá acotar de forma más exacta el tiempo y por consecuencia los costes del trabajo, así como el instrumental necesario.

- Salida gráfica final. Estará en función de las representaciones necesarias tanto durante la fase de estudio como en la presentación final de los resultados obtenidos. Existe la posibilidad de representar el modelo tridimensional mediante una estructura alámbrica, en la cual, los elementos plasman exclusivamente por las líneas de sus contornos, esto hace que en las vistas perspectivas, estos contornos se superpongan unos con otros. La alternativa consiste en modelos sólidos en los que, además de los contornos, se incluyen las superficies que definen, de esta manera en las vistas perspectivas los objetos más cercanos ocultan a los más alejados obteniéndose representaciones realistas. Aún se dispone de otro tipo de modelo sólido, en el que no se representa sólo sus superficies visibles sino que los muros son elementos macizos, es decir, que el interior no se considera hueco sino relleno, esta característica es especialmente interesante cuando se realizan secciones, de todas formas, los programas de dibujo asistido por ordenador no suelen dar muchas facilidades para realizar y gestionar modelos macizos por lo que, normalmente, hay que limitarse a geometrías sencillas o el aumento de trabajo necesario para la confección del modelo lo convierte en inviable (figura 2).

-En tercer lugar, es necesaria una correcta organización y codificación de la información obtenida que permita extraer sólo los elementos de interés en cada análisis.
Para la captura de la información y, conjuntamente con la topografía, puede recurrirse a la fotogrametría. La fotogrametría permite obtener una documentación exhaustiva y, si se recurre a pares estereoscópicos, se dispone además de un modelo óptico tridimensional de los elementos fotografiados que es a su vez un excelente archivo temporal del estado del edificio. A partir de los modelos estereoscópicos se puede extraer tanta información como sea necesario, por lo que son adecuados cuando la densidad de información a extraer es muy grande, por ejemplo, para el despiece completo "piedra a piedra» de un paramento.

La información se extrae de forma vectorial, es decir, como modelo alámbrico, y si lo que se requiere es algún tipo de modelo sólido se ha de realizar un proceso posterior de edición que convierta los perímetros en superficies y éstas en elementos macizos.

El trabajo fotogramétrico lleva implícito el empleo de un instrumental específico que consta de cámaras métricas calibradas, además de material auxiliar como andamios o grúas que permite obtener las tomas con una geometría correcta. Sin embargo, no se puede eludir el uso de la topografía para relacionar las coordenadas de los modelos estereoscópicos entre sí en un sistema tridimensional único. Esta relación se realiza mediante un conjunto de puntos que aparecen fotografiados y de los cuales se conocen sus coordenadas en el sistema tridimensional del objeto. La combinación de topografía y fotogrametría hace que el resultado final obtenga una gran calidad y un alto grado de detalle.

En ocasiones, las superficies a representar son irregulares, por ejemplo, los restos de un castillo en ruinas, las piedras que forman un dolmen o, simplemente, la superficie del terreno donde se asienta el edificio. Para conseguir la correcta aproximación geométrica además de la representación visual de la irregularidad se recurre a dividir las super- 


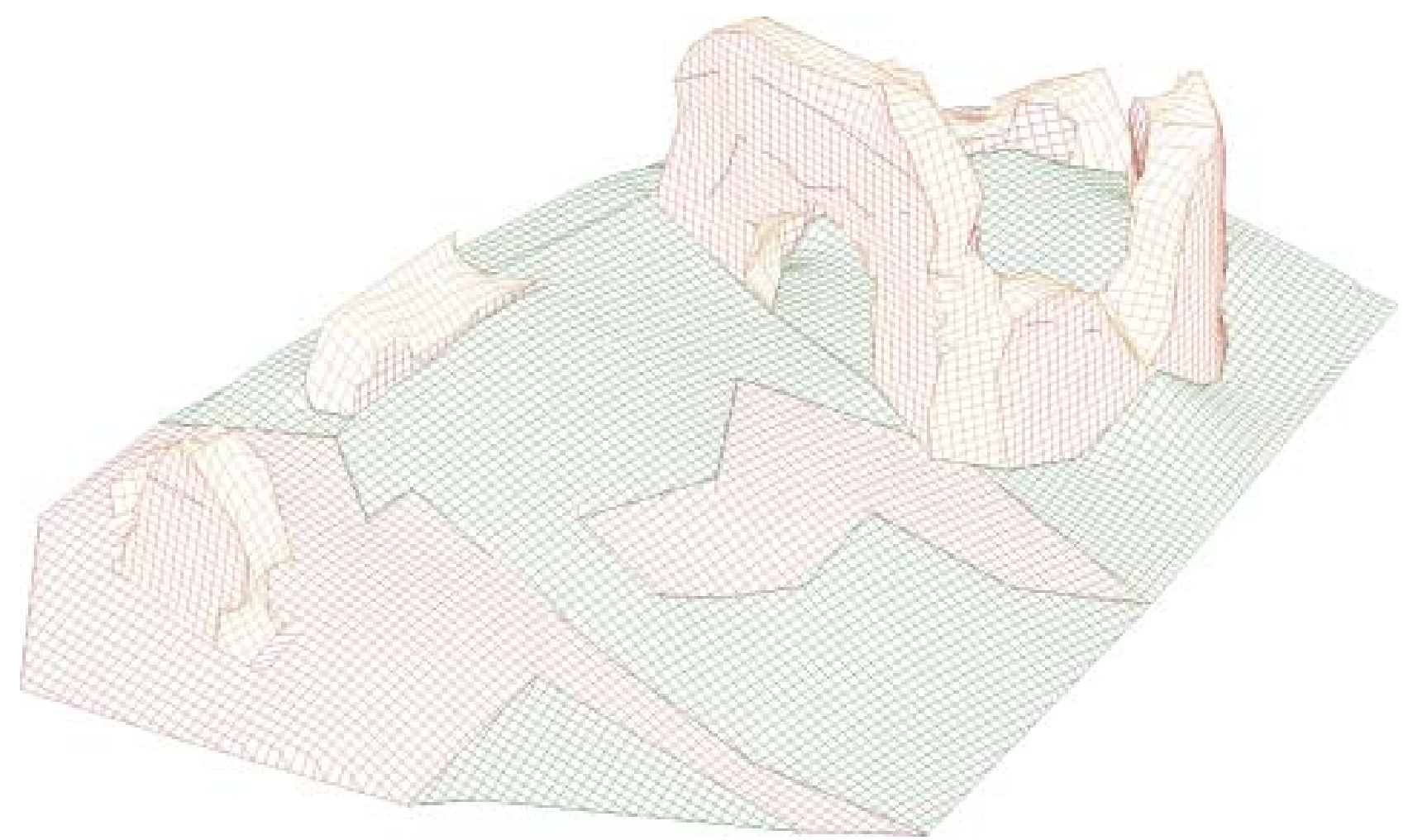

Fig. 3. Representación de superficies irregulares en mallas en el Castillo de Portilla (Álava)

ficies en teselas. Para ello, se parte del volumétrico previamente obtenido al que se añaden puntos de cota (figura 3 ).

Hasta aquí se ha considerado exclusivamente la información vectorial, pero también es posible incorporar la propia textura fotográfica como información métrica, para lo cual se proyectan las fotografías sobre el volumétrico corrigiéndolas del efecto perspectivo, obteniéndose así un modelo virtual. Los modelos virtuales son la puerta hacia las representaciones multimedia, en las cuales, se puede contar con maquetas realistas de los edificios a estudio que pueden analizarse interactivamente sirviendo además como vía de difusión del patrimonio tanto en su estado actual como en las reconstrucciones o recreaciones que se hagan de él.

El modelo virtual no es el único producto realizable a partir del volumétrico y las fotografías, al contrario, es posible obtener representaciones en las que se combine la información fotográfica con la restitución fotogramétrica o las superficies malladas. 